\title{
A review of exception reporting practices and understanding at Royal Bournemouth and Christchurch Hospitals NHS Foundation Trust
}

\author{
Authors: Ashley Hutchinson ${ }^{\mathrm{A}}$ and Stanley Tang ${ }^{\mathrm{A}}$
}

\section{Introduction}

Following the implementation of the 2016 junior doctor contract, exception reporting was introduced as a tool to improve junior doctor training, morale and patient safety by monitoring working hours. Thus far there has been minimal study into its effectiveness and acceptance in the NHS workforce. This audit assessed the use and views surrounding exception reporting at Royal Bournemouth and Christchurch Hospitals (RBCH).

\section{Materials and methods}

A SurveyMonkey questionnaire was circulated to 229 junior doctors (FY1-registrar) at RBCH. Questions related to hours worked and exception reporting habits over the last 2 months and their attitudes surrounding exception reporting.

\section{Results and discussion}

79 respondents from ten specialties were recorded. $83 \%$ of doctors indicated working additional hours, however only $35 \%$ of respondents had submitted an exception report. $63 \%$ were unaware they could report missed educational opportunities. $61 \%$ felt exception reporting would be viewed by seniors as a reflection of their competency and feared negative repercussions. $42 \%$ of respondents had been subject to/witnessed negative attitudes from seniors surrounding exception reports. Multiple barriers to reporting were highlighted including; unclear rota options on the reporting system, issues with account access, lack of training, as well as perceived negative senior attitudes towards exception reporting.

\section{Conclusion}

Despite being a potentially valuable asset in improving junior doctor rotas, training and patient safety, exception reporting is being significantly underutilised. This is likely due to a variety of factors, however most concerning is that of negative

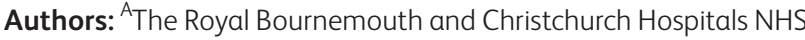
Foundation Trust, Bournemouth, UK attitudes and culture surrounding exception reporting. Addressing these issues will be crucial to increasing rates of exception reporting.

\section{Conflicts of interest}

None declared. 